\title{
Evaporation-driven Contact Angles in a Pure-vapor Atmosphere: the Effect of Vapor Pressure Non-uniformity
}

\author{
A.Y. Rednikov*, P. Colinet \\ Université Libre de Bruxelles, TIPs-Fluid Physics, 50 Av. F.D. Roosevelt \\ CP 165/67, 1050 Brussels, Belgium
}

\begin{abstract}
A small vicinity of a contact line, with well-defined (micro)scales (henceforth the "microstructure"), is studied theoretically for a system of a perfectly wetting liquid, its pure vapor and a superheated flat substrate. At one end, the microstructure terminates in a nonevaporating microfilm owing to the disjoining-pressure-induced Kelvin effect. At the other end, for motionless contact lines, it terminates in a constant film slope (apparent contact angle as seen on a larger scale), the angle being non-vanishing despite the perfect wetting due to an overall dynamic situation engendered by evaporation. Here we go one step beyond the standard onesided model by incorporating the effect of vapor pressure non-uniformity as caused by a locally intense evaporation flow, treated in the Stokes approximation. Thereby, the film dynamics is primarily affected through thermodynamics (shift of the local saturation temperature and evaporation rate), the direct mechanical impact being rather negligible. The resulting integrodifferential lubrication film equation is solved by handling the newly introduced effect (giving rise to the "integro" part) as a perturbation. In the ammonia (at $300 \mathrm{~K}$ ) example dealt with here, it proves to be rather weak indeed: the contact angle decreases while the integral evaporation flux increases just by a few percent for a superheat of $\sim 1 \mathrm{~K}$. However, the numbers grow (roughly linearly) with the superheat.
\end{abstract}

Keywords and phrases: contact angle, evaporation, superheat, pure vapor, thin films, nonlocal effect

Mathematics Subject Classification: 76D08, 76D45 80A22

\section{Introduction}

The present analysis belongs to the line of theoretical studies set forth by Potash and Wayner [9], formalized in a more rigorous way by Moosman and Homsy [6], and further developed in [1,7,11,13] (and references therein). Perhaps a key feature of this approach is accounting for the Kelvin effect due to both disjoining (Derjaguin) and Laplace pressures, important in a small vicinity (here generally referred to with the prefix 'micro-') of the contact line, and altering the local saturation temperature. In particular, with the disjoining pressure law appropriate for perfectly wetting non-polar liquids used in those studies, this

*Corresponding author. E-mail: aredniko@ulb.ac.be 


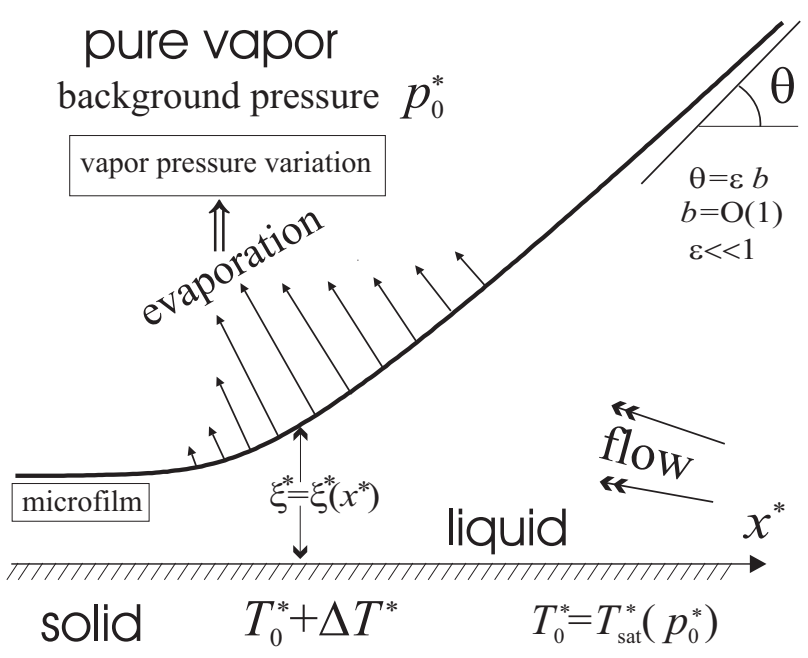

Figure 1. Configuration sketch.

implies that the contact line microstructure ends up in a non-evaporating microfilm covering the areas of the solid surface not occupied by "macroscopic" liquid samples, the microfilm being in thermodynamic equilibrium with the vapor at the temperature of the solid (higher than the saturation temperature corresponding to the vapor pressure). Otherwise, the studies mentioned above are essentially based upon the one-sided model by Burelbach et al. [3] of an evaporating liquid layer in contact with its pure vapor, whose noteworthy feature is accounting for a finite rate of evaporation kinetics (departure from local phase equilibrium at the liquid-vapor interface) which can get important on small scales.

In the steady case (meaning a contact line at rest, although a relatively intense flow, here referred to as the microflow, never ceases in its close vicinity due to the evaporation process), the microzone admits a film configuration asymptotically reaching a constant slope at distances much greater than the microscales (Stephan and Busse [13]). As the macroscopic curvature is small on the microscale, this configuration approximates to leading order the genuine film behavior in the microzone, with the final slope representing the apparent contact angle as seen on greater scales [10]. Thus, the microstructure problem gets decoupled, to leading order, from what happens at the macroscale. Such a decoupled problem is considered in its own right in $[7,11]$, and is also the subject of the present analysis (see Figure 1 for a sketch). Note also that the results thereby obtained are also valid for moving contact lines provided that the velocity of their motion is much smaller than the corresponding microflow scales, which are typically rather high (e.g. for ammonia at $300 \mathrm{~K}$ and a superheat of $\sim 1 \mathrm{~K}$ these may be of the order of hundreds of centimeters per second).

The evaporation-caused (micro)flow in the vapor induces a local pressure non-uniformity therein, neglected within the one-sided model. As a mechanical force, as far as the film behavior is concerned, this non-uniformity is most certainly negligible as compared to the disjoining and the Laplace pressures dominating in the microzone. Nonetheless, thermodynamically, as far as the local saturation temperature (and thus the local evaporation rate) is concerned, its importance relative to (the Kelvin effect of) the latter two gets augmented by a large factor given by the liquid-to-vapor density ratio. The evaporation process is a key factor in shaping the film profile, and it is in this way that the vapor pressure nonuniformity might exert its influence. Studying this effect is the goal of the present work. 


\section{Analysis}

The sketch of the contact line microstructure to be considered here is shown in Figure 1 . The wall/substrate is assumed to be smooth and flat on the corresponding scale. The problem belongs to the planar geometry. To the left, we have a microfilm in thermodynamic equilibrium with the vapor at the temperature of the solid $T_{w}^{*}=T_{0}^{*}+\Delta T^{*}$, which is higher (superheat $\Delta T^{*}>0$ ) than the saturation temperature $T_{0}^{*}=T_{\text {sat }}^{*}\left(p_{0}^{*}\right)$ corresponding to the background vapor pressure $p_{0}^{*}$. For the disjoining pressure versus the film thickness $\xi^{*}$ in the form $\Pi^{*}\left(\xi^{*}\right)=A^{*} / \xi^{* 3}$ with $A^{*}>0$ typical for perfectly wetting non-polar liquids, the microfilm thickness is

$$
\xi_{f}^{*}=\left(A^{*} / \Delta p^{*}\right)^{1 / 3} .
$$

It comes from the equality $\Pi^{*}\left(\xi_{f}^{*}\right)=\Delta p^{*}$, where

$$
\Delta p^{*}=\mathcal{L}^{*} \rho_{l}^{*} \Delta T^{*} / T_{0}^{*}
$$

is the pressure drop in the liquid required to have the equilibrium at $p_{v}^{*}=p_{0}^{*}, p_{l}^{*}=p_{0}^{*}-\Delta p^{*}$ and the temperature $T_{0}^{*}+\Delta T^{*} \cdot \mathcal{L}^{*}$ is the latent heat of evaporation, $\rho^{*}$ is the density. Hereafter the subscripts ' $v$ ' and ' $l$ ' refer to the vapor and the liquid, respectively. The above expression for $\Delta p^{*}$ is readily obtained by means of linearization of the chemical potential equality $\mu_{l}^{*}\left(T_{0}^{*}+\Delta T^{*}, p_{0}^{*}-\Delta p^{*}\right)=\mu_{v}^{*}\left(T_{0}^{*}+\Delta T^{*}, p_{0}^{*}\right)$, implying sufficiently small values of $\Delta T^{*}$. Here note that $\mu_{l}^{*}\left(T_{0}^{*}, p_{0}^{*}\right)=\mu_{v}^{*}\left(T_{0}^{*}, p_{0}^{*}\right)$ by the definition of $T_{0}^{*}$.

The dynamics of the liquid film is treated using the lubrication approximation $[2,8]$. The mass conservation reads

$$
\frac{\partial \xi^{*}}{\partial t^{*}}+\frac{\partial q^{*}}{\partial x^{*}}+\frac{j^{*}}{\rho_{l}^{*}}=0
$$

where $q^{*}$ is the volume flux in the $x^{*}$ direction, integrated over the cross-section of the film, and $j^{*}$ is the evaporation mass flux (per unit area of the film). Assuming the no-slip condition at the bottom and neglecting the dynamic influence of the gas phase, one has $[2,8]$

$$
q^{*}=-\frac{\xi^{* 3}}{3 \eta_{l}^{*}} \frac{\partial \tilde{p}_{l}^{*}}{\partial x^{*}}
$$

where $\eta^{*}$ is the dynamic viscosity. The pressure in the liquid is determined by the Laplace and disjoining pressure contributions (gravity is negligible at the scale of the contact-line microstructure):

$$
\tilde{p}_{l}^{*}=-\frac{A^{*}}{\xi^{* 3}}-\gamma^{*} \frac{\partial^{2} \xi^{*}}{\partial x^{* 2}},
$$

where $\gamma^{*}$ is the liquid-vapor interfacial tension. Hereafter, the tilde is used to denote the pressure deviation from the background pressure $p_{0}^{*}$ :

$$
\tilde{p}^{*}=p^{*}-p_{0}^{*}
$$

(for both the liquid and the vapor phases).

The expression for the evaporation flux $j^{*}$ is obtained from the following considerations. On the one hand, we have

$$
j^{*}=\frac{\lambda_{l}^{*}}{\mathcal{L}^{*}} \frac{T_{w}^{*}-T_{\Sigma}^{*}}{\xi^{*}},
$$

as given by heat conduction through the liquid layer (neglecting that through the vapor) still on account of the lubrication (here better to say, thin-film) approximation. Here $\lambda^{*}$ is the thermal conductivity, and $T_{\Sigma}^{*}$ is the temperature at the liquid-vapor interface (generally varying along the film). On the other hand, we have a kinetic law (Hertz-Knudsen-Schrage-like [12])

$$
j^{*}=\frac{L_{w w}^{*} \mathcal{L}^{*}}{T_{0}^{* 2}}\left(T_{\Sigma}^{*}-T_{0, \text { loc }}^{*}\right)
$$


written assuming a small temperature difference on the right-hand side relative to $T_{0}^{*}$, with the kineticgas-theory expression for $L_{w w}^{*}$ given by

$$
L_{w w}^{*}=\frac{f_{a}}{2-f_{a}} \rho_{v}^{*} \sqrt{\frac{2 M_{w}^{*} T_{0}^{*}}{\pi R^{*}}},
$$

where $f_{a}$ is a phenomenological accommodation coefficient, $R^{*}$ is the universal gas constant, $M_{w}^{*}$ is the molecular weight, and $\rho_{v}^{*}=p_{0}^{*} M_{w}^{*} / R^{*} T_{0}^{*}$ is the saturated vapor density (at temperature $T_{0}^{*}$ and pressure $\left.p_{0}^{*}\right) . T_{0, \text { loc }}^{*}$ is the local saturation temperature, generally varying along the liquid-vapor interface. It is different from $T_{0}^{*}$ on account of, on the one hand, the Kelvin effect (owing to the pressure jump between the vapor and the liquid), and on the other hand, the vapor pressure locally deviating from $p_{0}^{*}$ due to the evaporation flow in the vapor phase. Recurring to the linearization of the chemical-potential equality similar to the derivation of $(2.1)$, one obtains

$$
T_{0, \text { loc }}^{*}-T_{0}^{*}=\frac{T_{0}^{*}}{\mathcal{L}^{*} \rho_{l}^{*}}\left(-\tilde{p}_{l}^{*}+\frac{\rho_{l}^{*}}{\rho_{v}^{*}} \tilde{p}_{v}^{*}\right) .
$$

It is accounting for such a vapor pressure non-uniformity that represents the novel contribution of the present paper. This effect has never been treated before in the studies of evaporation near contact lines. Assuming that the two contributions in (2.8) are of the same order of magnitude, whereas $\rho_{l}^{*} / \rho_{v}^{*} \gg 1$, it is consistent to neglect $\tilde{p}_{v}^{*}$ versus $\tilde{p}_{l}^{*}$, which we in fact did in (2.4). In other words, the excess pressure in the liquid $\tilde{p}_{l}^{*}$ is determined just by the disjoining and the Laplace pressures, whereas any excess normal stress contribution into $\tilde{p}_{l}^{*}$ from the vapor side is neglected. Thus, we neglect the direct mechanical impact of $\tilde{p}_{v}^{*}$ on the liquid film while keeping the thermodynamical one in (2.8) on account of $\rho_{l}^{*} / \rho_{v}^{*} \gg 1$.

Combining (2.5), (2.6) and (2.8), one can obtain

$$
j^{*}=\frac{\lambda_{l}^{*}}{\mathcal{L}^{*}}\left[\Delta T^{*}+\frac{T_{0}^{*}}{\mathcal{L}^{*} \rho_{l}^{*}}\left(\tilde{p}_{l}^{*}-\frac{\rho_{l}^{*}}{\rho_{v}^{*}} \tilde{p}_{v \Sigma}^{*}\right)\right]\left[\xi^{*}+\frac{\lambda_{l}^{*} T_{0}^{* 2}}{L_{w w}^{*} \mathcal{L}^{* 2}}\right]^{-1},
$$

where the subscript ' $\Sigma$ ' is added to underscore that $\tilde{p}_{v}^{*}$ is evaluated at the interface (in the liquid, $\tilde{p}_{l}^{*}$ is uniform across the film to leading order in the lubrication approximation). We note that, in the absence of the Kelvin and vapor pressure non-uniformity effects, we arrive in (2.9) at an expression similar to the one used in [3].

Finally, the film evolution equation is given by (2.2) with (2.3), (2.4), (2.7) and (2.9). But in fact, one closure (an additional relation on $\tilde{p}_{v \Sigma}^{*}$ ) is still needed in order to finalize the formulation. We shall consider it after rendering the problem dimensionless. From now on, we shall imply a steady problem $\left(\partial / \partial t^{*} \equiv 0\right)$.

Hereafter, we shall use the notation

$$
f^{*}=[f] f,
$$

where $f$ (without asterisk) is the dimensionless version of a dimensional quantity $f^{*}$ (with asterisk), $[f]$ being the scale. The microfilm thickness provides a natural film-thickness scale in the present microstructure problem: $[\xi]=\xi_{f}^{*}$. The scale $[x]$ is then chosen from the considerations (apart from numerical factors introduced into the scaling for historical reasons and arguably for convenience) that the two contributions in (2.4) are of the same order: $[x]=\xi_{f}^{* 2} / \sqrt{3} a^{*}$, where $a^{*}=\left(A^{*} / \gamma^{*}\right)^{1 / 2}$ is a molecular length scale $[4,5]$. The lubrication approximation implies that

$$
\epsilon=\frac{[\xi]}{[x]}=\frac{\sqrt{3} a^{*}}{\xi_{f}^{*}} \ll 1,
$$

which is also a condition for the microfilm to still be describable in the framework of continuum mechanics $\left(\xi_{f}^{*} \gg a^{*}\right)$. The evaporation flux scale is chosen based on heat conduction across the film: $[j]=\lambda_{l}^{*} \Delta T^{*} / \mathcal{L}^{*} \xi_{f}^{*}$. The vapor pressure variation scale is taken to correspond to the evaporation-induced 
Stokes flow: $\left[\tilde{p}_{v}\right]=\eta_{v}^{*}\left([j] / \rho_{v}^{*}\right)[x]^{-1}$. Finally, the dimensionless steady lubrication equation for the film thickness $\xi=\xi(x)$ can be written as

$$
\left(\xi^{3} \xi^{\prime \prime \prime}-\xi^{\prime} / \xi\right)^{\prime}+E j=0
$$

with

$$
j=\frac{1-1 / \xi^{3}-3 \xi^{\prime \prime}-\alpha \tilde{p}_{v \Sigma}}{\xi+K},
$$

where the prime denotes the derivative with respect to $x$. The dimensionless numbers are

$$
E=\frac{\eta_{l}^{*} \lambda_{l}^{*} T_{0}^{*}}{3 a^{* 2}\left(\mathcal{L}^{*} \rho_{l}^{*}\right)^{2}}, K=\frac{2-f_{a}}{f_{a}} \sqrt{\frac{\pi R^{*} T_{0}^{*}}{2 M_{w}^{*}}} \frac{\lambda_{l}^{*} T_{0}^{*}}{\rho_{v}^{*} \mathcal{L}^{* 2} \xi_{f}^{*}}
$$

and

$$
\alpha=\left(\frac{\rho_{l}^{*}}{\rho_{v}^{*}}\right)^{2} \frac{\eta_{v}^{*}}{\eta_{l}^{*}} E \epsilon^{3} .
$$

Generally, we imply $E=O(1)$ and $K=O(1)$, unlike $\epsilon \ll 1$. $E$ is referred to as the evaporation number. $K$ is the kinetic resistance number (a consequence of accounting for a finite-rate evaporation kinetics, $K=0$ would correspond to local phase equilibrium at the interface). In the expression for $\alpha$, note that even though $\epsilon$ is assumed to be small, $\alpha$ can in principle be $O(1)$ owing to the first factor therein typically being large for a liquid-vapor system. The standard one-sided model is recovered at $\alpha=0$, when, up to notations and scaling factors and excluding other additional physical effects sometimes incorporated into the model, Eq.(2.10) becomes of course the same as elsewhere (cf. $[1,7,10,11,13])$.

The microstructure boundary conditions are

$$
\xi=1 \quad \text { as } x \rightarrow-\infty, \quad \xi \sim b x \quad \text { as } x \rightarrow+\infty,
$$

i.e. to the left we have the non-evaporating microfilm, while to the right a constant-slope behavior. Note that $b=O(1)$ is a rescaled contact angle, the genuine one being

$$
\theta=\epsilon b,
$$

i.e. $\theta \ll 1$ together with $\epsilon \ll 1$. The rescaled angle $b=b(E, K, \alpha)$ is an eigenvalue of the resulting boundary-value problem. It is expected that $\tilde{p}_{v_{\Sigma}} \rightarrow 0$ as $x \rightarrow \pm \infty$ (cf. below), i.e. the vapor pressure non-uniformity is localized within the microstructure and the problem is still well posed with the boundary conditions (2.11).

As already mentioned, an additional relation involving $\tilde{p}_{v \Sigma}$ is needed in order to close the problem. This comes from considering the evaporation flow in the vapor phase. In doing so, on account of $\epsilon \ll 1$, the interface is treated just as flat, and the problem for the flow is formulated in the upper half-space. The dimensionless (scale $[j] / \rho_{v}^{*}$ ) normal velocity at the boundary is specified by $j(x)$, whereas the tangential velocity is set equal to zero (no slip) given that the velocity in the vapor is expected to be much greater than that in the liquid. The velocity field fades away at infinity. It is assumed that the Reynolds number, $[j][x] / \eta_{v}^{*}$, is small so that the Stokes approximation can be used. The following result can be derived (e.g. by recurring to a Fourier integral in the $x$ direction, see Appendix A for more details):

$$
j(x)=-\frac{1}{2 \pi} \int_{-\infty}^{+\infty} \tilde{p}_{v_{\Sigma}}(\bar{x}) \log |\bar{x}-x| d \bar{x},
$$

or its inverse

$$
\tilde{p}_{v \Sigma}(x)=\frac{2}{\pi} \int_{-\infty}^{+\infty} j^{\prime \prime}(\bar{x}) \log |\bar{x}-x| d \bar{x}
$$


which provide the sought closure to (2.10). As it could be expected, the effect of the vapor pressure non-uniformity is nonlocal with regard to the film, resulting in an integro-differential character of the problem (2.10), (2.12). A noteworthy feature of the resulting vapor flow field is that it happens to exert no tangential stress on the boundary (see Appendix A), hence no mechanical impact on the film in this regard either.

With (2.11) and the assumption (confirmed below) that $\tilde{p}_{v \Sigma}$ fades away at infinity, we have $j \sim 1 /(b x)$ as $x \rightarrow+\infty$. Another assumption (also readily confirmed below) is that $j$ decreases faster than $1 / x$ as $x \rightarrow-\infty$. Then one can deduce the following asymptotic behavior from Eq.(2.12b):

$$
\tilde{p}_{v \Sigma} \sim-\frac{2}{\pi x^{2}}\left[\frac{\log |x|-1}{b}+\int_{-\infty}^{1} j(\bar{x}) d \bar{x}+\int_{1}^{+\infty}\left(j(\bar{x})-\frac{1}{b \bar{x}}\right) d \bar{x}\right] \quad \text { as } \quad x \rightarrow \pm \infty,
$$

confirming the conjectures on $\tilde{p}_{v_{\Sigma}}$. In view of (2.13), a few leading-order terms of the film thickness behavior at infinity actually remain of the same form as in the absence of the effect $(\alpha=0)[11]$ :

$$
\xi=b\left(x-x_{0}\right)-\frac{E}{4 b^{4}} \log ^{2} \frac{x}{k}+O\left(\frac{\log ^{3} x}{x}\right) \text { as } x \rightarrow+\infty,
$$

where $b=b(E, K, \alpha), k=k(E, K, \alpha)$ are to be obtained by solving the boundary-value problem (2.10)(2.12), while $x_{0}$ is present due to the invariance of the problem to shifts along $x$. The same is true for the asymptotics of the integral flux $J(x)$. We have

$$
J(x) \equiv \int_{-\infty}^{x} j(\bar{x}) d \bar{x}=-(1 / E)\left(\xi^{3} \xi^{\prime \prime \prime}-\xi^{\prime} / \xi\right)
$$

the latter equality being written on account of (2.10a). Hence,

$$
J=\frac{1}{b}\left(\log \frac{x}{k}-\frac{3}{2}\right)+O\left(\frac{\log ^{2} x}{x}\right) \text { as } x \rightarrow+\infty,
$$

as found earlier for $\alpha=0$ [11]. The scale is $[J]=[j][x]=\lambda_{l}^{*} \Delta T^{*} / \mathcal{L}^{*} \epsilon$. As $x \rightarrow-\infty$, however, an exponential approaching the microfilm taking place for $\alpha=0$ is now replaced by an algebraic law: $\xi=1+O\left(\log |x| / x^{2}\right)$, and the same is true for the local flux: $j=O\left(\log |x| / x^{4}\right)$ (the latter confirming one of the earlier made conjectures). Note that, using the parameter $k=k(E, K, \alpha)$, the result (2.13) can be rewritten in the form

$$
\tilde{p}_{v \Sigma} \sim-\frac{2 \log (|x| / k)-5}{\pi b x^{2}} \text { as } x \rightarrow \pm \infty .
$$

In the present paper, we limit ourselves to considering the vapor pressure non-uniformity effect as a perturbation (although a possible numerical method for solving the problem in full is detailed in Appendix B). In this scheme, the leading-order solution formally corresponds to $\alpha=0$ and is well investigated (see e.g. [11] for an extensive parametric study). The leading-order distribution $j=j(x)$ thereby obtained is subsequently used in (2.12) to calculate the distribution $\tilde{p}_{v \Sigma}=\tilde{p}_{v \Sigma}(x)$. This latter now appears as an inhomogeneity in the linearized (around the leading-order solution) version of Eq.(2.10) used for the first correction. In this way, the result for the rescaled contact angle in particular gets represented in the form $b=b_{0}(E, K)+\alpha b_{1}(E, K)$.

Consider the case of ammonia at $T_{0}^{*}=300 \mathrm{~K}$ as an example [13]. The values used for estimation are $\rho_{l}^{*}=600 \mathrm{~kg} / \mathrm{m}^{3}, \rho_{v}^{*}=9 \mathrm{~kg} / \mathrm{m}^{3}, \nu_{l}^{*}=\eta_{l}^{*} / \rho_{l}^{*}=2.2 \times 10^{-7} \mathrm{~m}^{2} / \mathrm{s}, \nu_{v}^{*}=\eta_{v}^{*} / \rho_{v}^{*}=1.2 \times 10^{-6} \mathrm{~m}^{2} / \mathrm{s}, f_{a}=1$, $A^{*}=2 \times 10^{-21} \mathrm{~J}, M_{w}^{*}=0.017 \mathrm{~kg} / \mathrm{mol}, \gamma^{*}=0.02 \mathrm{~N} / \mathrm{m}, \mathcal{L}^{*}=1.18 \times 10^{6} \mathrm{~J} / \mathrm{kg}, \lambda_{l}^{*}=0.48 \mathrm{~W} / \mathrm{m} \mathrm{K}$. For $\Delta T^{*}=1 \mathrm{~K}$, one obtains $\epsilon=0.58, E=0.124, K=5.74$ and $\alpha=9$. Figs. 2 and 3 show the results for $j=j(x)$ (leading order) and $\tilde{p}_{v \Sigma}=\tilde{p}_{v \Sigma}(x)$. The fact that the numerical values of $j$ and $\tilde{p}_{v \Sigma}$ turn 


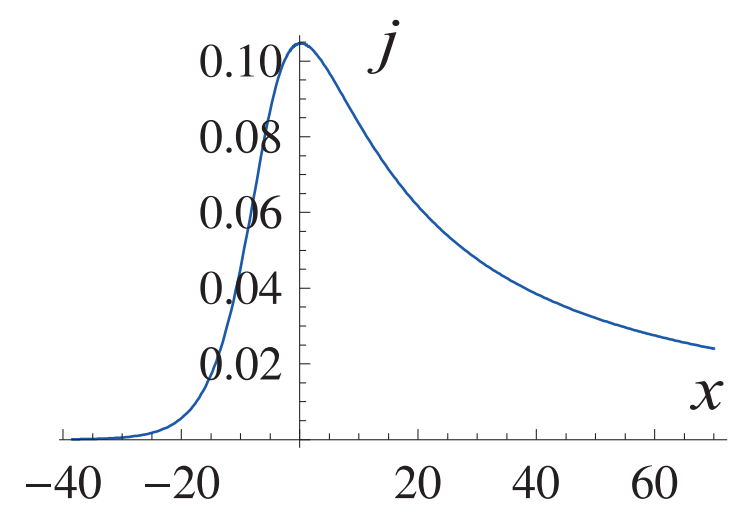

FiguRE 2. Local flux distribution for $\alpha=0$.

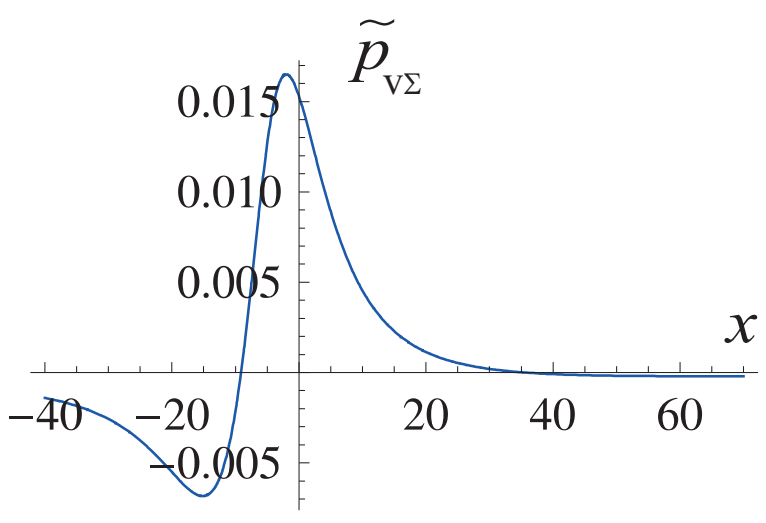

FiguRE 3. Excess pressure in the vapor along the interface.

out to be relatively small inspires confidence that the case $\alpha=9$ is still amenable to the perturbation approach. Besides, note that $p_{v \Sigma}(x)$ changes sign, in accordance with the property (A3), which must somehow mitigate the overall effect. The computation yields $b=0.573-0.0010 \alpha$, which shows indeed that for $\alpha=9$ the result is modified just by a couple of percent. The dimensionless number $\alpha$ can be seen to be proportional to the superheat, so that for larger superheats (say, for $\Delta T^{*} \sim 10 \mathrm{~K}$ when $\alpha \sim 90$ ) the effect is expected to be much more significant, even though the perturbation approach is most certainly no longer valid in this case. On the other hand, note that the case of ammonia considered above is characterized by quite a large value of the saturation pressure, $p_{0}^{*} \sim 10.5 \mathrm{bar}$. As a consequence, the vapor density is rather large, while the vapor kinematic viscosity is rather small, so that the property ratios entering the definition of $\alpha$ are not so large. For smaller saturation pressures (and accordingly smaller $T_{0}^{*}$ ), one can expect much greater values of $\alpha$ owing to the property ratios. But on the other hand, the number $K$ also tends to increase with $\Delta T^{*}$ and as $p_{0}^{*}$ decreases, leading to smaller $j$ and larger effective scales along $x$ (see [11]) and thus (as it can be conjectured) tending to reduce the effect of vapor pressure non-uniformity. A further parametric study is needed and other examples should be considered in order to fully clarify its importance. The present work just introduces the effect and points that in certain cases it may probably be quite significant. 


\section{Concluding Remarks}

An additional physical effect affecting the microstructure of a contact line of a perfectly wetting liquid overlaid with its pure vapor on a superheated substrate has been considered. It is neglected in the classical one-sided model and, excluding the possibility of a breakdown of the continuum approach in the vapor phase on the microscale, it is apparently the most serious factor to potentially undermine the one-sidedness. The effect in question is the vapor pressure non-uniformity owing to an intense evaporation in the microstructure zone and the associated vapor flow. We have argued that this mechanism enters principally in a thermodynamic way (its direct mechanical impact being much less important), by shifting the local saturation temperature at the film surface and thus affecting the evaporation rate, the evaporation being the key factor leading to a finite apparent contact angle. It is the latter most important characteristic of the microstructure that we have focused upon in the present study.

Overall, we have found that the studied effect is not strong enough to undermine the classical approach. At least, it tends to be weaker than what dimensional estimations may indicate. One of the reasons is that the dimensional scales may eventually get modified by large/small numerical factors, a point upon which we insisted in [11]. Another reason is that the vapor pressure non-uniformity changes sign within the microstructure, thus reducing the global impact. But on the other hand, the effect is nevertheless appreciable enough to warrant further attention, all the more so that its importance grows with the superheat and might turn out to be much more significant for some fluids.

As for the vapor flow resulting in the pressure non-uniformity in question, we have found the Stokes (small Reynolds number) approximation to be good enough for its description, even though the tendency at sufficiently large superheats is towards the Reynolds numbers of order unity.

Mathematically, we have dealt with a lubrication equation for the liquid film where the newly incorporated effect enters by means of a nonlocal operator. Such type of mathematical model (lubrication equation + nonlocal operator) can be envisaged to be of more general interest in the problem of the contact line microstructure, e.g. when considering the effects of a finite thermal conductivity of the substrate or of an inert-gas admixture in the vapor. A noteworthy feature of the effect considered here is its localization within the microstructure: it turns out to decay fast enough away from the microstructure core.

Acknowledgements. Financial support from ESA \& BELSPO PRODEX projects is gratefully acknowledged, together with the support of the Marie Curie ITN Network MULTIFLOW. Senior Research Associate PC also gratefully acknowledges financial support of the Fonds de la Recherche Scientifique - FNRS.

\section{References}

[1] V.S. Ajaev. Spreading of thin volatile liquid droplets on uniformly heated surfaces. J. Fluid Mech., 528 (2005), $279-296$.

[2] D. Bonn, J. Eggers, J. Indekeu, J. Meunier, E. Rolley. Wetting and spreading. Rev. Mod. Phys., 81 (2009), $739-805$.

[3] J.P. Burelbach, S.G. Bankoff, S.H. Davis. Nonlinear stability of evaporating/condensing liquid films. J. Fluid Mech., 195 (1988), 463-494.

[4] P.G. de Gennes. Wetting: statics and dynamics. Rev. Mod. Phys., 57 (1985), 827-863.

[5] P.G. de Gennes, F. Brochard-Wyart, D. Quéré. Capillarity and wetting phenomena. Springer, 2004.

[6] S. Moosman, G.M. Homsy. Evaporating menisci of wetting fluids. J. Colloid Interface Sci., 73 (1980), $212-223$.

[7] S.J.S. Morris. Contact angles for evaporating liquids predicted and compared with existing experiments. J. Fluid Mech., $432(2001), 1-30$.

[8] A. Oron, S.H. Davis, S.G. Bankoff. Long-scale evolution of thin liquid films. Rev. Mod. Phys., 69 (1997), 931-980.

[9] M. Potash, P.C. Wayner. Evaporation from a two dimensional extended meniscus. Int. J. Heat Mass Transfer, 15 (1972), 1851-1863.

[10] A.Ye. Rednikov, P. Colinet. Vapor-liquid steady meniscus at a superheated wall: asymptotics in an intermediate zone near the contact line. Microgravity Sci. Tech. 22 (2010), 249-255.

[11] A.Ye. Rednikov, S. Rossomme, P. Colinet. Steady microstructure of a contact line for a liquid on a heated surface overlaid with its pure vapor: parametric study for a classical model. Multiphase Sci. Tech., 21 (2009), 213-248.

[12] R.W. Schrage. A Theoretical Study of Interface Mass Transfer. Columbia University Press, New York, 1953. 
[13] P.C. Stephan, C.A. Busse. Analysis of the heat transfer coefficient of grooved heat pipe evaporator walls. Int. J. Heat Mass Transfer, 35 (1992), 383-391.

\section{Appendix A: Evaporation-Induced Stokes Flow in the Vapor}

Here we consider the flow in the vapor phase as caused by the evaporation from the liquid film within the microstructure region. This is a two-dimensional planar flow. Let $x$ and $z$ be the Cartesian coordinates, where $x$ is along the solid surface, as before, whereas $z$ is orthogonal to it. We use the scales $[z]=[x]$, where $[x]$ is the longitudinal length scale of the microstructure defined in Section 2. Let $u_{v}$ and $w_{v}$ be the corresponding velocity components of the vapor flow $\left(\left[u_{v}\right]=\left[w_{v}\right]=[j] / \rho_{v}^{*}\right)$, and $\psi_{v}$ the stream function $\left(u_{v}=\partial \psi_{v} / \partial z, w_{v}=-\partial \psi_{v} / \partial x,\left[\psi_{v}\right]=[j][x] / \rho_{v}^{*}\right)$. Even though the velocity (micro)scale $[j] / \rho_{v}^{*}$ can be rather large, the length scale $[x]$ of the microstructure is yet so small that the resulting Reynolds number, $R e_{v}=[j][x] / \eta_{v}^{*}=[J] / \eta_{v}^{*}$, is considered to be small. Therefore, the flow in question is treated in the Stokes approximation. Furthermore, consistently with the lubrication (thin-film) approximation used for the treatment of the liquid film, we neglect to leading order the $z$ extent of the film, thus imposing the evaporation-flux boundary condition just at $z=0$. We assume that, due to a large value of the density ratio $\rho_{l}^{*} / \rho_{v}^{*}$, the velocity in the vapor phase is much greater than that in the liquid film, and thus we impose a no-slip condition at $z=0$ for the tangential component of the velocity field. Finally, the flow is assumed to fade away at infinity.

With all these assumptions, the problem for the vapor flow is given by the Stokes equations (here in terms of the stream function, while the pressure variable $\tilde{p}_{v}$ is defined as in Section 2)

$$
\left(\frac{\partial^{2}}{\partial x^{2}}+\frac{\partial^{2}}{\partial z^{2}}\right)^{2} \psi_{v}=0, \quad \frac{\partial \tilde{p}_{v}}{\partial x}=\left(\frac{\partial^{2}}{\partial x^{2}}+\frac{\partial^{2}}{\partial z^{2}}\right) \frac{\partial \psi_{v}}{\partial z}
$$

at $z>0$, and the boundary conditions

$$
\psi_{v} / \sqrt{x^{2}+z^{2}} \rightarrow 0, \quad \tilde{p}_{v} \rightarrow 0
$$

as $\sqrt{x^{2}+z^{2}} \rightarrow \infty(z>0)$, and

$$
-\frac{\partial \psi_{v}}{\partial x}=j(x), \quad \frac{\partial \psi_{v}}{\partial z}=0
$$

at $z=0$.

The solution is readily obtained in terms of the Fourier components:

$$
f_{q}=(2 \pi)^{-1} \int_{-\infty}^{\infty} f(x) e^{-i q x} d x, \quad f(x)=\int_{-\infty}^{\infty} f_{q} e^{i q x} d x
$$

for a typical quantity $f$. Thus, one obtains

$$
\psi_{v q}=i q^{-1}(1+|q| z) e^{-|q| z} j_{q}, \quad \tilde{p}_{v q}=2|q| e^{-|q| z} j_{q},
$$

and in particular the most important result here

$$
\tilde{p}_{v \Sigma q}=2|q| j_{q}
$$

where the subscript ' $\Sigma$ ' refers to evaluation at $z=0$.

We note that, since the integral flux (2.15) diverges logarithmically as $x \rightarrow+\infty$, the transform $j_{q}$ must diverge logarithmically as $q \rightarrow 0$. Then, on account of (A2), $\tilde{p}_{v \Sigma q} \rightarrow 0$ as $q \rightarrow 0$, and thus

$$
\int_{-\infty}^{\infty} \tilde{p}_{v_{\Sigma}}(x) d x=0
$$


We note also that $-i \operatorname{sign}(q) / 2$ is the Fourier transform of $1 / x$, whereas $|q|=q \operatorname{sign}(q)$. Therefore, in terms of the original variables, the relation (A2) can be rewritten in the form of a convolution with the function $1 / x$ as follows:

$$
j^{\prime}(x)=-\frac{1}{2 \pi} \int_{-\infty}^{\infty} \frac{\tilde{p}_{v \Sigma}(\bar{x})}{x-\bar{x}} d \bar{x}
$$

or rather

$$
\tilde{p}_{v \Sigma}(x)=\frac{2}{\pi} \int_{-\infty}^{\infty} \frac{j^{\prime}(\bar{x})}{x-\bar{x}} d \bar{x},
$$

where the integrals are understood in the sense of the principal value, the results being subsequently rendered in the form (2.12).

Another noteworthy feature of the considered Stokes flow in the vapor is that the tangential stress exerted by the flow on the boundary $z=0$, i.e. on the liquid film, turns out to vanish identically, even though it was the no-slip condition that we imposed. Indeed, the stress, appropriately non-dimensionalized, is given by $\partial u_{v} / \partial z+\partial w_{v} / \partial x=\partial^{2} \psi_{v} / \partial z^{2}-\partial^{2} \psi_{v} / \partial x^{2}$, or by $\partial^{2} \psi_{v q} / \partial z^{2}+q^{2} \psi_{v q}$ in terms of the Fourier transform, evaluated at $z=0$. Using (A1) in here yields zero. This is perfectly coherent with neglecting the mechanical influence of the flow in the vapor on the film dynamics as practiced in the present paper.

Finally, we estimate the vapor Reynolds number, $R e_{v}=[J] / \eta_{v}^{*}$ (see above, and the same for the definition of $[J]$ ), for the ammonia example considered at the end of Section 2 (with $T_{0}^{*}=300 \mathrm{~K}$ and $\left.\Delta T^{*}=1 \mathrm{~K}\right)$. We obtain $[x] \sim 1.6 \mathrm{~nm},[j] / \rho_{v}^{*} \sim 48 \mathrm{~m} / \mathrm{s}$, and thus $R e_{v} \sim 0.065$. For a more precise estimation of the vapor Reynolds-number regime, based not just on the dimensional scales but rather on the actual flow characteristics, one may notice from Figure 2 that $j_{\max } \sim 0.1$ and $\Delta x \sim 30$ for the maximum value of $j(x)$ and the typical width of the $j(x)$ distribution, respectively. This brings in an additional factor of the order of $j_{\max } \Delta x \sim 3$ and thus raises the vapor Reynolds number estimation to 0.2 , nevertheless still remaining small. We see that the Stokes approximation for the vapor flow is a good one indeed. On the other hand, $R e_{v}$ must increase with the superheat $\Delta T^{*}$ (even though slower than linearly) and the case of a finite $R e_{v}$ is also potentially of interest. We note in addition that the consideration of the vapor recoil effect sometimes encountered in the literature (e.g. [11]) makes sense as such and can be carried out within a one-sided model just in the opposite limit to the one studied here: $R e_{v} \gg 1$. In this case, the vapor recoil, of the order of $[j]^{2} / \rho_{v}^{*}$, is much greater than the vapor pressure non-uniformity. Otherwise, for e.g. $R e_{v}=O(1)$, they are of the same order, and thus the vapor recoil cannot be treated separately from the vapor flow. For $R e_{v} \ll 1$, it becomes negligible. Furthermore, note once again that a direct mechanical influence on the film has been neglected here even as far as the pressure non-uniformity is concerned and we have claimed that it affects the film behavior primarily thermodynamically through the evaporation rate, whereas such a thermodynamical role of the vapor recoil is limited to the Kelvin effect and is thus $\rho_{v}^{*} / \rho_{l}^{*}$ times relatively as weaker as that of the pressure non-uniformity. Let us also mention that the vapor velocity scale $[j] / \rho_{v}^{*}$ estimated above for the ammonia example, even though seemingly large, is still essentially smaller than the typical scale $\sqrt{R^{*} T_{0}^{*} / M_{w}^{*}} \sim 400 \mathrm{~m} / \mathrm{s}$ of the sound speed, hence justifying treating the vapor as incompressible. This becomes even more justified taking into account the numerical factor $j_{\max } \sim 0.1$ pointed out above. Nonetheless, in particular since the vapor velocity increases with the superheat, the vapor compressibility can potentially become an issue and it would not be unwise to monitor the corresponding criterion for each particular example considered.

\section{Appendix B: A Possible Numerical Method}

Apart from the purely practical purpose, pointing out a numerical method can also be useful, in the absence of a rigorous mathematical proof, from the viewpoint of making reasonably sure that the problem 
is well posed. This is all the more so for the problem under consideration because here the usual lubrication type of formulation gets mingled with nonlocal (integral) operators.

The boundary-value problem (2.10)-(2.12) is formulated in an infinite interval $-\infty<x<+\infty$. In a simplest numerical method, this gets approximated by $x_{\min } \leq x \leq x_{\max }$, where $x_{\max }$ and $x_{\min }$ are sufficiently large positive and negative values, respectively. Let us introduce $N$ grid points $n=1,2, \ldots N$ (say, uniformly spaced) in the interval $x_{\min } \leq x \leq x_{\max }$. Then the numerical problem contains $3 N$ unknowns: the values of $\xi, j$ and $\tilde{p}_{v \Sigma}$ on the grid.

The fourth-order differential equation (2.10a) can straightforwardly be discretized (say, using finite differences) in the points $n=3,4, \ldots N-2$, hence already $N-4$ equations. Another 4 equations come from satisfying boundary conditions (2.11), posed at $x=x_{\max }$ and $x=x_{\min }$ within the numerical scheme. Namely, $\xi=1$ and $\xi^{\prime \prime}=0$ at $x=x_{\min }$, and $\xi=\xi_{\max }$ and $\xi^{\prime \prime}=0$ at $x=x_{\max }$ (where the second derivative is meant to be appropriately discretized). Thus, $N$ equations so far, coming from (2.10a) and (2.11).

A tricky point though is the value of $\xi_{\max }$ : it must be large enough (just as $x_{\max }$ and $x_{\min }$ ), but not too large, while otherwise can be arbitrary. Indeed, the solution must not depend on $\xi_{\max }$ (within the numerical precision) in the sense that different values of $\xi_{\max }$ at $x=x_{\max }$ must correspond to the same solution just shifted along the $x$ axis (note that the problem (2.10)-(2.12) is invariant to shifts along $x$ ). At the same time, it is not difficult to figure out the reason for the mentioned limitations by looking at the expected structure of the film profile (cf. Figure 1): if $\xi_{\max }$ is too large, there will be too much of the "wedge" part in the interval $x_{\min } \leq x \leq x_{\max }$, whereas if $\xi_{\max }$ is not large enough, there will be too much of the microfilm part. It would be reasonable to avoid these extremes.

Eq.(2.10b) can straightforwardly be discretized in the internal points $n=2,3, \ldots N-1$. It is not less straightforward in the end points $n=1$ and $n=N$ once the boundary conditions $\xi^{\prime \prime}=0$ are once again invoked. In the integral equation $(2.12 \mathrm{a})$, the integration limits are now from $x_{\min }$ to $x_{\max }$, and it can obviously be discretized at all the points $n=1,2, \ldots N$.

Thus, all in all, we obtain $3 N$ equations (some of them nonlinear) for $3 N$ unknowns, which can be solved iteratively e.g. by means of the Newton-Raphson method. The rescaled contact angle of the microstructure, $b$, is then obtained simply by calculating $\xi^{\prime}$ at $x=x_{\max }$ (cf. (2.11)).

A further refinement of the numerical method must be possible with the use of the asymptotic behaviors established in this paper, viz. (2.14), (2.16), (2.17), etc., permitting to improve the precision without increasing the values of $x_{\max }$ and $\left|x_{\min }\right|$. 